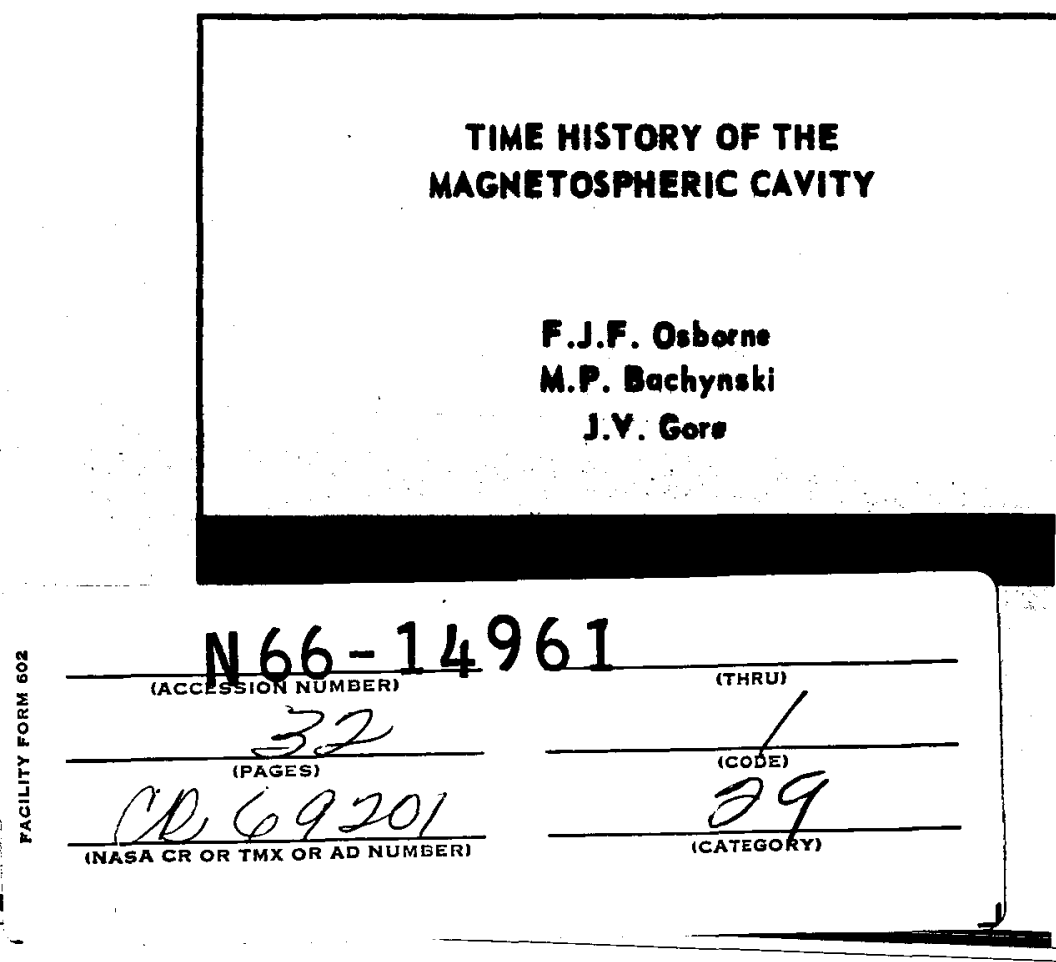

JUNE 1965

Prepared Under

Contract PD69-400007, Sorial 2PD4-5

of the

DEFENCE RESEARCH BOARD OF CAMADA
GPO PRICE \$

CFSTI PRICE(S) \$

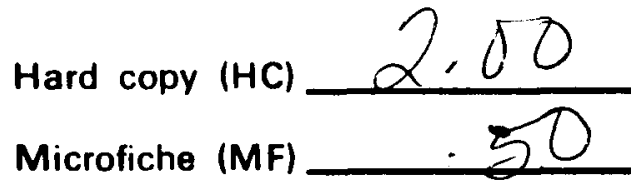

f 653 July 65

\author{
and \\ NASA Controct MAS $_{w} 1088$ \\ RCA VICTOR COMPANY, LTD. \\ RESEARCH LABORATORIES \\ MONTREAL, CANADA
}




\section{TIME HISTORY OF THE MAGNETOSPHIBRIC CAVITY}

\section{F.J.F. Osborne, M.P. Bachynski, J.V. Gore RCA Victor Research Laboratories Montreal, Canada}

$$
\text { - ABSTRACT - }
$$

$$
14961
$$

Scale model laboratory experiments simulating the effect of the solar wind on the magnetosphere have show the time history of the formation and breakup of the magnetospheric cavity. Time resolved ( 1 usec) data on the visible "standoff" interaction show that the "quasi Van Allen belts" first become visible well within the cavity at the time the plasma wind encounters the fringing terrella field. The belts at this time show a definite structure with "ribbons" of plasma along the field lines. This structure is probably associated with oscillatory phenomena present in the belts and their excitation mechanism. The polar views show considerable asymmetry of both the internal and external phenomena throughout the interaction. The visual asymmetries indicate that the plasma in the tail of the dipole magnetic field "wags" as the interaction builds up and decays. Magnetic field measurements in the equatorial plane show a similar motion of the magnetic field in the tail. Awthos

* Paper presented URSI Spring leeting, Washington, D.C. (1965). 


\section{INTRODUCTION}

A strong interaction occurs when the solar wind or plasma ejected from the sun streams into interplanetary space and encounters the magnetic field of the earth. This interaction results in a distortion of the earth's magnetic field both in space and at the earth's surface (magnetic storm) and numerous other complex natural phenomena. The complex nature of the interaction makes it extremely difficult to sort out the crucial mechanisms contributing to the numerous observed effects.

In an effort to try to get a better understanding of some of the phenomena involved in order to account for the observations made both by space craft and from the earth resort has been made to laboratory experiments based on scaled models. (Osborme et al 1963, 1964, 1964a, 1964b). The experiments consist of propelling a stream of plasma with a plasma gun into the vieinity of a three-dimensional dipole magnetic field and studying the interaction. Previous results of such experiments in which plasma has been shot at the equatorial plane of the magnetic field have shown:

(i) a stand-off of plasma at approximately the position where the kinetic pressure of the solar wind is balanced by the magnetic pressure of the dipole magnetic field

(ii) the sweeping of the magnetic field and the formation of the front side of the magnetospheric cavity (in agreement with space measurements)

(iii) the formation of trapped regions of plasma similar in shape and position to the natural Van Allen belts, and which exhibit oscillatory phenomena (Osborne et al 1964) 
(iv) weakly trapped regions of plasma in the vicinity of the polar regions

(v) the motion of the "dip" pole under the influence of the plasma

(vi) a westward motion of the plasma in the simulated Van Allen belts

(vii) the dependence of penetration of plasma into the polar regions on the properties of the solar wind

This paper, after considering various aspects of the relevant parameters involved in the scaling presents new results showing the time history of the formation and decay of the magnetospherie carity and associated phenomena under the action of "gust" of solar wind.

\section{CONSIDERATIONS OF SOME RELEVANT PARAMETERS}

The major considerations of the scaling requirements pertaining to a model experiment on the interaction between the solar wind and the magnetsphere have been considered previously (Shkarofsky (1962), Bachynski (1964) and Shkarofsky (to be published)). It seems worthwhile however, to treat in detail the relative magnitudes of some of the parameters involved as well as several other key aspects. This section $\mathrm{wlll}$ therefore be devoted to several such topies namely the cyclotron radius, the magnetospheric boundary at the equatorial plane and the Debye length.

\section{(a) The Cyclotron Radius}

The cyclotron radius $\left(r_{b}\right)$ for a charged particle gyrating in a magnetic field (B) is given by:

$$
r_{b}=\frac{v_{t}}{\omega_{b}}=5.68 \times 10^{-8} \nabla_{t} m_{0} / \mathrm{B} \mathrm{cm}
$$


where $v_{t}$ is the thermal velocity of the particle in $\mathrm{cm} / \mathrm{sec}$ $\omega_{b}$ is the cyclotron frequency for the given particle mo is the mass of the particle in electron mass unito i.e. $m_{0}=1$ for an electron, $m_{0}=1836.6$ for a proton, ete.

$B$ is the magnetic field in gauss.

The variation of cyclotron radius with magnetic field strength, thermal velocity and mass (species) is shown in Figure 1. The regime of space parameters and laboratory parameters are indicated in the Figure. In relation to the current model experiments, typical values of the cyclotron radius for a typical thermal velocity of $10^{5} \mathrm{~cm} / \mathrm{sec}$ are given in Table I.

As can be seen, the electron cyclotron radius is exceedingly small. Previous experiments show from spectroscopic data that oxygen ions are trapped within the cavity. Here the magnetic fields are in the kilogauss range corresponding to an ion eyclotron radius of a few millimeters. Hence "mirroring" of the charged particles trapped in the dipole field of the terrelle should take place. At the boundary of the magnetospheric carity, the incoming plasma is seeded with barium ions with a resulting cyclotron radius of several centimeters. However, as shown in the next section, the boundary thickness is determined more by the electrons than the fons and therefore this relatively large ion cyclotron radius has a secondary effect on the scaling.

\section{(b) The Boundary}

(i) The Sheath Thickness

The main features of one model of an idealized plasma-magnetic field interface has been considered among others by Ferraro (1952), Rosenbluth (1963), Grad (1961), Colgate (1961), Scarf (1964) and Sesters (1965). In this simple two-dimensional case, one can consider plasma streaming at right angles to a magnetic field (see Fig.2). This can be considered analogous to the solar wind 
being incident along the equatorial plane where the earth's magnetic field is transverse to the flow. The main features of the particle trajectories are discussed in the aforementioned references and will only be summerized here. The fons with their larger mass (and hence greater kinetic energy) tend to penetrate further into the magnetic field than the electrons. This charge separation gives rise to an electric field $\left(\mathrm{F}_{\mathrm{g}}\right)$ which accelerates the electrons in the $\mathrm{E}_{8} \times \bar{B}$ direction and therefore causes them to execute broader turns. Simaltaneously, the ions lose energy until they are finally turned around quite sharply. In fact, considerations of conservation of particle energy and momentum require that the ions and electrons exchange energy momentarily, but regain their original energies as they leave the interface or sheath. Thus the lons determine the depth of penetretion but the electric field due to charge separation determines the final particle trajectories. (This streaming of electrons as found in the boundary sheath is known to give rise to instabilities. However, dissipation of energy due to collisional effects or the fact that the magnetospheric boundary is curved not planar, may present the growth of suoh instabilities.)

The different depths of penetration of the electrons and lons results in a transition region or sheath of thickness $\delta$ where:

$$
\delta=c / \omega_{p}=5.32 \times 10^{5} / \sqrt{n}_{\mathrm{n}} \mathrm{cm}
$$

where $c$ is the velocity of light

${ }^{\omega} p$ is the plasma frequency

$n$ is the charge particle number density $\left(\mathrm{cm}^{-3}\right)$

Thus in the simple case the sheath thickness depends solely on the charge particle number density.

Equation (2) can be put in several different forms. Thus for a thermal, non-streaming plasma, the balance conditions between the thermal pressure and magnetic pressure require that:

$$
n K T=B^{2} / 2 \mu_{0}
$$


where $x$ is Boltzmann's constant

$T$ is the electron temperature $\left({ }^{\circ} \mathrm{K}\right)$

B is the magnetic field

$\mu_{0}$ is the permeability of free space

Using the fact that the electron thermal veloeity $\nabla_{t}=\left(2 \mathrm{kr} / \mathrm{m}_{E}\right)^{\frac{1}{2}}$ where $\mathrm{m}_{\theta}$ is the electron mass, we can write:

$$
\delta=\nabla_{t} / w_{b}=3.38 v^{\frac{1}{2}} / B \text { om }
$$

( $V$ is the electron temperature in electron volts, $B$ is magnetic field in gauss )

Thus we see that the sheath thickness for a thermal non-streaming plasma is of the order of the electron eyelotron radius.

For a streaming plasma (streaming velocity considerably exceeds the thermal velocity), the balance conditions require that: (considering specular reflection at the boundary)

$$
2 n\left(m_{1}+m_{e}\right) \nabla_{0}^{2}=B^{2} / 2 \mu_{0}
$$

where: $m_{i}$ is the ion mass

Vo is the streaming velocity.

Using this expression, the sheath thickness can be written:

$$
\delta=\frac{\sqrt{2} \nabla_{0}}{\omega_{b e} \frac{m_{0}}{m_{1}}} .
$$

where: $\omega_{b e}=\frac{\theta B}{m_{\theta}}$ the electron cyelotron frequency.

Typical values for the parameters at the magnetospheric boundary are: $B \sim 10^{-3}$ gauss, $\nabla_{0} \sim 500 \mathrm{~km} / \mathrm{sec}, \mathrm{n} \sim 1-10$ particles $/ \mathrm{cm}^{3}$, ions are protons so that $\sqrt{m_{\theta} / m_{i}} \sim \frac{1}{42.8}$. This gives typical sheath thickness estimates of:

$$
\delta \sim 1-5 \mathrm{~km}
$$


(A mose complete sheath theory (Scarf (1964)) which considers fluctuating fields and hot electrons in the sheath give sheath thicknesses of the order of $70 \mathrm{~km}$. )

Typical laboratory values are: B 50-500 gauss 'at boundary, $\nabla_{0} \sim 2 \times 10^{6} \mathrm{~cm} / \mathrm{sec}, \mathrm{n} \sim 10^{12}-10^{13}$ particles $/ \mathrm{cm}^{3}$, lons are barium (atomie weight 136). This leads to sheath thickness values of

$$
\delta \sim 0.1-1 \mathrm{~cm}
$$

As reported previously (Osborne et al 1964b) magnetic boundaries as thin as 3 ma have been observed experimentally.

(ii) Boundary Postion,

Equation (5)gives the magnetic field value $B$ at the stand-off position and can be exressed as:

$$
n A V_{0}^{2}=1.18 \times 10^{22} B^{2}
$$

where: $A$ is the mass number of the ion speoies and $\mathrm{n}$ is in particles/ $\mathrm{cm}^{3}$, $\nabla_{0}$ in $\mathrm{c} / \mathrm{sec}$ and $\mathrm{B}$ in gauss. Normelly the magnetic field. $B_{0}$ at any location ro on the equatorial plane varies in a dipole fashion namely:

$$
\mathrm{B}_{0}=\mathrm{B}_{\mathrm{E}}\left(\frac{\mathrm{R}_{\mathrm{T}}}{\mathrm{r}_{0}}\right)^{3}
$$

where: $B_{B}$ and $R_{B}$ are the magnetic field values and radius at the equator respectively.

Due to the streaming plasma, the magnetic field at the location $r_{0}$ will be increased from its value $B_{0}$ to some value $\alpha B_{0}$. If we assume that the field is still approximately dipole in configuration the field at the boundary position (B) or equivalently the location of the boundary position ( $I_{0}$ ) can be determined by considering a dipole field whose field value at the equator has been increased by a value $a$, i.e.

$$
\frac{r_{0}}{B_{E}}=\left(\frac{\alpha B_{E}}{B}\right)^{1 / 3}
$$


where: $a$ is the factor by which the unperturbed field at position $r_{0}$ has been increased at the stand-off position (usually $\alpha \sim 2$ ).

From Equation (8) and (7)

$$
\frac{r_{0}}{R_{E}}=\left[\frac{\left(\frac{\alpha B}{E}\right)^{2}}{2 \mu_{0}} \frac{1}{2 n m_{1} \nabla_{0}^{2}}\right]^{1 / 6}
$$

In the natural situation, taking $n \sim 5 / \mathrm{cm}^{3}, \nabla_{0}=500 \mathrm{~km} / \mathrm{sec}$, $\alpha \sim 2$, protons, $B_{E}=0.312$ gauss jields: $B \sim 10^{-3}$ gauss, $x_{0} / R_{B} \sim 8.5$.

In the laboratory $\alpha, \nabla_{0}, r_{0} / R_{F}, B_{B}$ and $B$ ean be measured directly (0sborne ot al $(1964 b)$ ). Hence Equation (7) can be used to determine the electron density and Bquation (8) as a check on the boundary position. A typical velue is: $B=250$ gauss, $A=136, V_{0}=2 \times 10^{6} \mathrm{ar} / \mathrm{sec}$. This leads to $n \sim 10^{12}$ particles/ $\mathrm{cm}^{3}$. The boundary positions agree within the accuracy to which it is possible to measure $\alpha$ and $B$.

\section{(c) Debre Iength}

An indication of the charge separation effects is given by the Debye length $\left(\lambda_{D}\right)$, namely:

$$
\lambda_{\mathrm{D}}=0.69 \sqrt{\mathrm{T} / \mathrm{n}} \text { en }
$$

Typical Iaboratory values are: $\left(n=10^{11}-10^{13} / 0 \mathrm{~m}^{3}, T \sim 30,000^{\circ}\right)$

$$
\lambda_{\mathrm{D}} \sim 10^{-4}-10^{-5} \mathrm{~cm}
$$

In space: $\left(n \sim 10^{\circ}-10 / \mathrm{cm}^{3}, T \sim 1,000^{\circ}\right)$

$$
\lambda_{D} \sim .1-1 \mathrm{~m}
$$

\section{(d) Discussion}

of pertinence to the scaling is a comparison of the ratios of various parameters in the natural situation to those in the laboratory. In relationship to the thickness of the magnetospheric boundary a dimensionless parameter which may be of importance is the sheath thickness compared to the ion cyclotron 
radius $\left(r_{b i}\right)$ hairor an ion thermal veloeity of $v_{f}$ we have:

$$
\frac{\text { sheath thickness }}{\text { ion cyclotron radius }}=\delta / r_{b i}=\frac{e}{\omega_{p}} \cdot \frac{\omega_{b i}}{\nabla_{t}}
$$

A typieal laboratory value: $\nabla_{t} \sim 2 \times 10^{5} \mathrm{om} / \mathrm{sec}, n=10^{12} / \mathrm{cm}^{3}, m_{1}=136$ times the proton mass, B 200 gauss at the boundary.

For these parameters: $\delta / r_{b i} \cong 0.02$

For a magnetic field inereased by a factor $10, \delta / r_{b 1} \sim 0.2$ [Values in the laboratory can range from 0.01 to 10.]

In spece if we take $\mathrm{T}_{t} \sim 10^{\mathrm{em}} / \mathrm{see}, \mathrm{n} \sim 4 / \mathrm{cm}^{3}, \mathrm{~m}_{1}=$ mass of proton, $B \sim 10^{-3}$ gauss

$$
\delta / r_{b i} \sim 0.025
$$

[Values in space can range over 0.01 to 100]

Thus in both cases this ratio is not that far different. (The use of

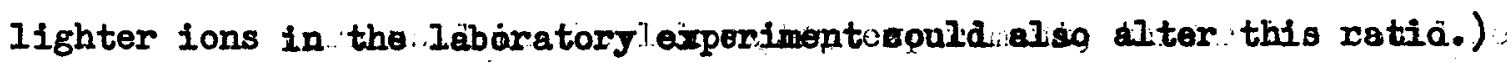

We also note that both the ion cyolotron radius and the sheath thickness are many Debye lengths in extent. This is true both in space and in the laboratory. It is therefore evident that similar mechanisms may be operative in the laboratory scale model experiments as in the natural situation: TIMG HISTORY OF MAGNETOSPHERE

The time history of the formation and decay of the magnetospherio cavity under the action of a "gust" of solar wind is illustrated in a series of photographs taken with a 1 microsecond exposure at predetermined times after the launching of the plasma simulating the solar wind. Figure 3 illustrates the plasma formation as viewed from the sunrise equatorial position. The views from above the polar axis are shom in Figure 4. The measurements made at 
identicel times should be considered simulteneously in ascertaining the time bistory of the interaction between the solar wind plasma and the three-dimensional dipole of the model earth or terrella.

The important features of the buildup and collapse of the magnetospher cavity to note are:

1. With reference to Figures 3 and 4 it is apparent that $15 \mu$ sec after launching the plasma from the solar wind "gun" there is no visible plasma in the vicinity of the terrella. The first indication of plasma occurs at $17 \mu$ sec - a diffuse trapped region on the windward side of the terrella showing pronounced striations or periodic structure when seen from the polar axis. (Some plasma at this same time penetrates into the polar regions.) The situations show a periodicity of about $0.6 \mathrm{~cm}$ or wavelength of $1.2 \mathrm{~cm}$. Hence we can consider a disturbance with a wavelength of the order of a centimetre. Calculating the order of magnitude of the Alfren velocity $\nabla_{A}$ for these conditions,

$$
V_{A}=\frac{B}{\sqrt{\mu_{0} n m_{i}}}=\frac{2.2 \times 10^{11} \mathrm{~B}}{\sqrt{n A}} \mathrm{em} / \mathrm{sec}
$$

and taking: $B \sim 100$ gauss, $A=32, n \sim 10^{12} / \mathrm{cm}^{3}$ we arrive at

$$
\nabla_{A} \sim 4 \times 10^{6} \mathrm{~cm} / \mathrm{sec} .
$$

This would correspond to a disturbanee with:frequeney $f_{A}$ given by:

$$
f_{A}=\nabla_{A / \lambda_{A}}=4 \times 10^{6} / 1.2 \sim 3.3 \mu_{c} / \mathrm{sec}
$$

: It may be noted that this Alfren velocity is calculated for the position of the interior phenomena and will represent the highest velocity (highest B) experienced to this point by a wave initiated at the moving front, assuming that the density is constant over th rodume. 
Both electrical probe and magnetic probe measurements in the plasme in this region show pronounced signal fluctuations at discrete frequencies (Osborme et al 1964). The frequeney of these fluctuations are in the $100 \mathrm{Ke}$ to $1 \mathrm{Mc}$ range. It therefore appears that these fluctuations and striations could be due to a magnetohydrodynamio ware which is generated as the plasma first strikes the outer edge of the dipole field. (Although at these times the incoming plasma from the plasma gun is not visible in the field of vien, magnetic measurements show that this plasma has already struck the outer magnetic field of the terrella).

2. This interior region becomes better defined (indicating a definite trapping mechanism in the dipole magnetic fleld), shearing into two regions as seen from the poles) diffusing the striations and exhibiting a distinct westward motion. (The diffusing of the striations may be due to the fact that the incoming plasma continues to move against the dipole magnetic field and thus various magnetohydrodynamic wave modes may be excited so that no single periodicity is now possible.) This plasma whose distribution resembles that of the Van Allen belts in space is formed entirely by precursor radiation from the plasma gun at a time considerably in advance of the main stream of plasma wich only becomes visible at $23 \mu \mathrm{sec}$ from the time of launching. 3. The solar wind plasma as $1 t$ arrives, compresses the dipole field (see the 25 and $27 \mu$ sec photographs) moving the magnetospherio boundary and the Van Allen belt position nearer towards the terrella. At 33 to $39 \mu \mathrm{sec}$, equilibrium appears to have been achieved after which time the solar wind plasma decreases in pressure. 
4. Regions of plasma become trapped in the polar regions (in addition to the simulated Van Allen belts) in the interaction times 29-45 $\mu$ sec. As seen from the polar view, this plasma forms a "tail" trying to encircle the terrella (in addition to the westward motion with increasing time of the interaction).

5. At about the $45 \mu$ sec time, the solar wind plasma eases its pressure. This is accompanied by a very dramatic change in the plasma trapped in the vicinity of the terrella. The plasma originally trapped in the polar regions is now absent. The plasma in the pagnetospheric cavity begins to break up into a complicated structure. There is a precipitation of plasma into the polar regions in a ring structure around the dip pole (note particularly the 51 usee polar view).

6. At late times ( $>54 \mu \mathrm{sec}$ ) the "belt" structure diffuses, leaving principally the plasma trapped in the simulated Van Allen belts which lasts for a long time (> 75 нsee).

Another feature which has been measured by different techniques is equally noteworthy. The component of the magnetic field measured in the equatorial plane at the time of maximum magnetic field compression ( $33 \mu s e c)$ is shown in Fig. 5. This plot shows a magnetic field "tail" of the magnetospheric cavity swung about $60^{\circ}$ westward from the wind direction in agreement with the visible plasma "tail" shown earlier. Since with very little solar wind pressure, the backside of the magnetosphere must be nearly directly behind the windward direction, this implies a "wag" of the magnetospheric tail during the interaction of the solar wind. The asymetry of the earth's magnetic field in the equatorial plane is also apparent from the above plot. Some indication of an asymmetry In the earth's magnetospheric cavity has been given by Obayashi (1964) although 
space data on this point are as yet very meagre. (Dessler and Walters (1964)) have discussed a "wag" of the magnetospherie tail but their mechanism is different than that which could be acting here.) SUDARAY

Detailed examination has been made of the processes occurring within the boundary region of a scaled interaction between the solar wind and the earth's magnetic dipole field. It has been shown that the pertinent parameters of Debye length, Rosenbluth sheath criterion $\left(c / \omega_{p}\right)$ and the ion oyclotron radii are of approximately the same relative magnitude for both the geophysieal and laboratory situations. Thus under similar plasma regimes, the same types of processes may be expected to occur.

Thereas in space, measurements of magnetospheric boundary thickness are complicated by boundary fluctuations and satellite motion, the laboratory measurements clearly indicate a Rosenbluth sheath mechanism rather than direct control by the ion eyolotron radias.

Time resolved data on the visible features of a "standoff" interaction show that the quasi Van Allen belts exhibit the first luminosity and are also of long persistence. The period ofi initigd gemetation bf these belts shows considerable structure. The periodicity of this structure in conjunotion with the oseillatory phenomena previously reported suggests: an wha water perturbation.

Regions of plasma nominally trapped within the magnetosphere in the region of the neutral points have been found to be precipitated out on the. terrella surface at specific times of the interaction and to appear on the surface as a ring of luminosity approximatily centered on the dip-poles. 
The polar view of the magnetosphere shows considerable, asymetry of both the internal and external phenomena. These asymmetries vary with time and thus generate a "rag" of the magnetospherio tail. These data are confirmed by magnetic measurements in the equatorial plane.

\section{AGKNOKILDGEMISTSS}

This work was done under Contract PD69-400007 Serial 2PD4 -5 of the Defence Research Board of Canada and NASA Contract NAST 1088. 
RHFERENCES

Bachynaki, M.P. (1964), simulation of geophysical phenomena in the laboratory, AIAA Jour. 2 1873-1882.

Colgate, S.A. (1961), Ioniration in orossed electric and magnetic fields in Blectromagnetics and Fuid Iynomics of Gaseous Elasma, ed. J.Fox, Polytechnic Press, N.Y. 1961, 373-386.

Dessler, A.J. and G.K. Walters (1964), Hydromagnetic coupling between solar wind and magnetosphere, Planet Space Sci. 12, 227-234.

Ferraro, V.C.A. (1952), On the theory of the first phase of a geomagnetic storm: a new illustrated calculation based on an idealized (plane not cylindrical) model field distribution. J.Geophys.Res. 57, 15.

Grad, H. (1961), Boundary layer between a plasma and a magnetic field, Pays. Pluids 4, 1366-1375.

Obayashi, T. (1964), Magnetosphere and its boundary, Helport of Ionosphere and Space Research in Japan XVIII, No.3, 228-234.

Osborne, F.J.F., I.P. Shkarofsky and J.V. Gore (1963), Iaboratory simulation of the solar wind magnetosphere interaction, Can.J.Phys. 41, 1747-1752.

Osborne, F.J.P., M.P. Bachynski and J.V. Gore (1964), Oscillatory phenomena in the laboratory simulation of the magnetosphere. Symposium on Ultra Low Frequency Blectromagnetic Fields. Boulder, Colarado, Aug.17-20. Osborne, F.J.F., M.P. Bachynski and J.V. Gore (1964a), Laboratory measurements of plasma trapping within the magnetosphere, Appl.Physiletters 2, 77-79. Osborne, F.J.P., K.P. Bachynski and J.V. Gore (1964b), Laboratory studies of the variation of the magnetosphere with solar wind properties. J.Geophys. Res. 69, $4441-4449$.

Rosenbluth, 4 . (1963), Surface layer model in the limit of no collisions, Plasma Physics and Thermonuclear Research, J.L. Tuck, W.B. Thompson, editors, Pergamon Press Ine. Iondon. 271-277. 
Scarf, F.I. (1964), The solar wind and its interaction with magnetic fields, Space Physies, D.P. LeGalley, A.Rosen editors, J. Wiley and Sons Ine. New York 437-473.

Sestero, A. (1965), Charge separation effects in the Ferraro-Rosenbluth cold plasma sheath model, Phys.Fluids 8 739-744.

Shkarofsky, I.P. (1962), The solar wind, the magnetosphere and their simulation in the laboratory, RCA Victor Company, Research Report 7-801-22.

Shkarofsky, I.P. (To be published), Laboratory simulation of disturbances produced by bodies moving through a plasma and of other geophysical phenomena. Astronautica Acta. 


\begin{tabular}{|l|l|l|l|}
\hline B (gauss) & $r_{b}$ (electrons) & $r_{b}\left(0_{2}\right.$ ion) & $r_{b}$ (B ion) \\
\hline $\begin{array}{l}\text { 1,000 inside } \\
\text { magnetosphere }\end{array}$ & $2.8 \times 10^{-5} \mathrm{~cm}$ & $1.7 \mathrm{~cm}$ & $7.1 \mathrm{~cm}$ \\
& $5.7 \times 10^{-6} \mathrm{~cm}$ & $0.33 \mathrm{~cm}$ & $1.4 \mathrm{~cm}$ \\
\hline
\end{tabular}

Table I 


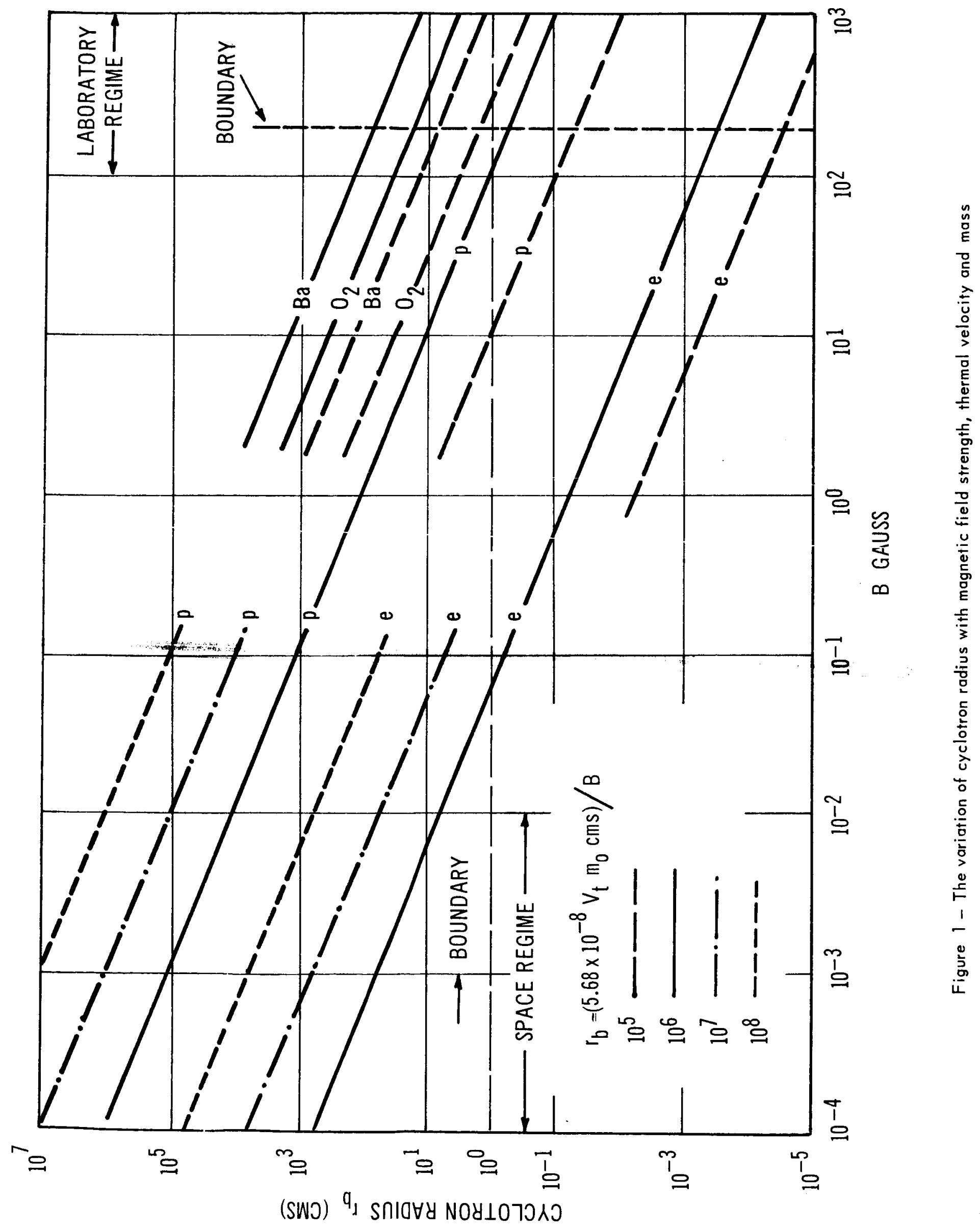




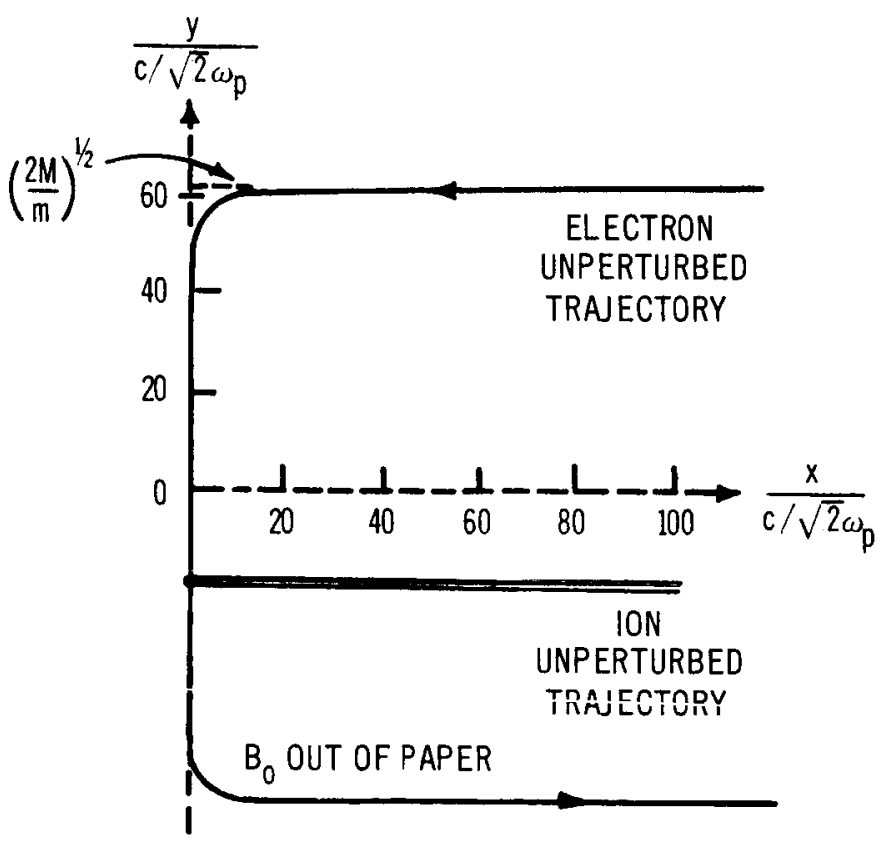

Figure 2 - The idealized electron and trajectories in a plasmo-magnetic field interface 

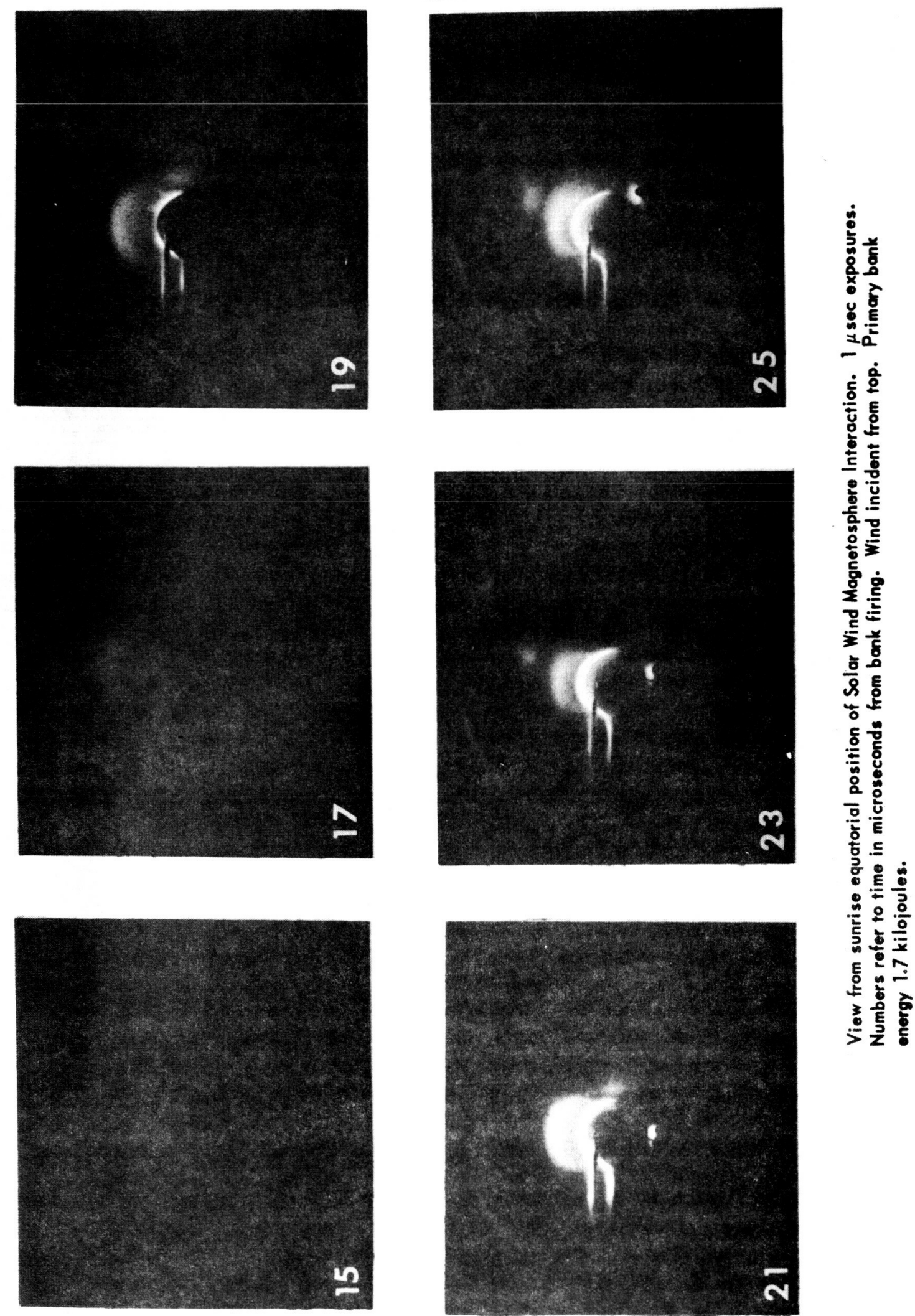

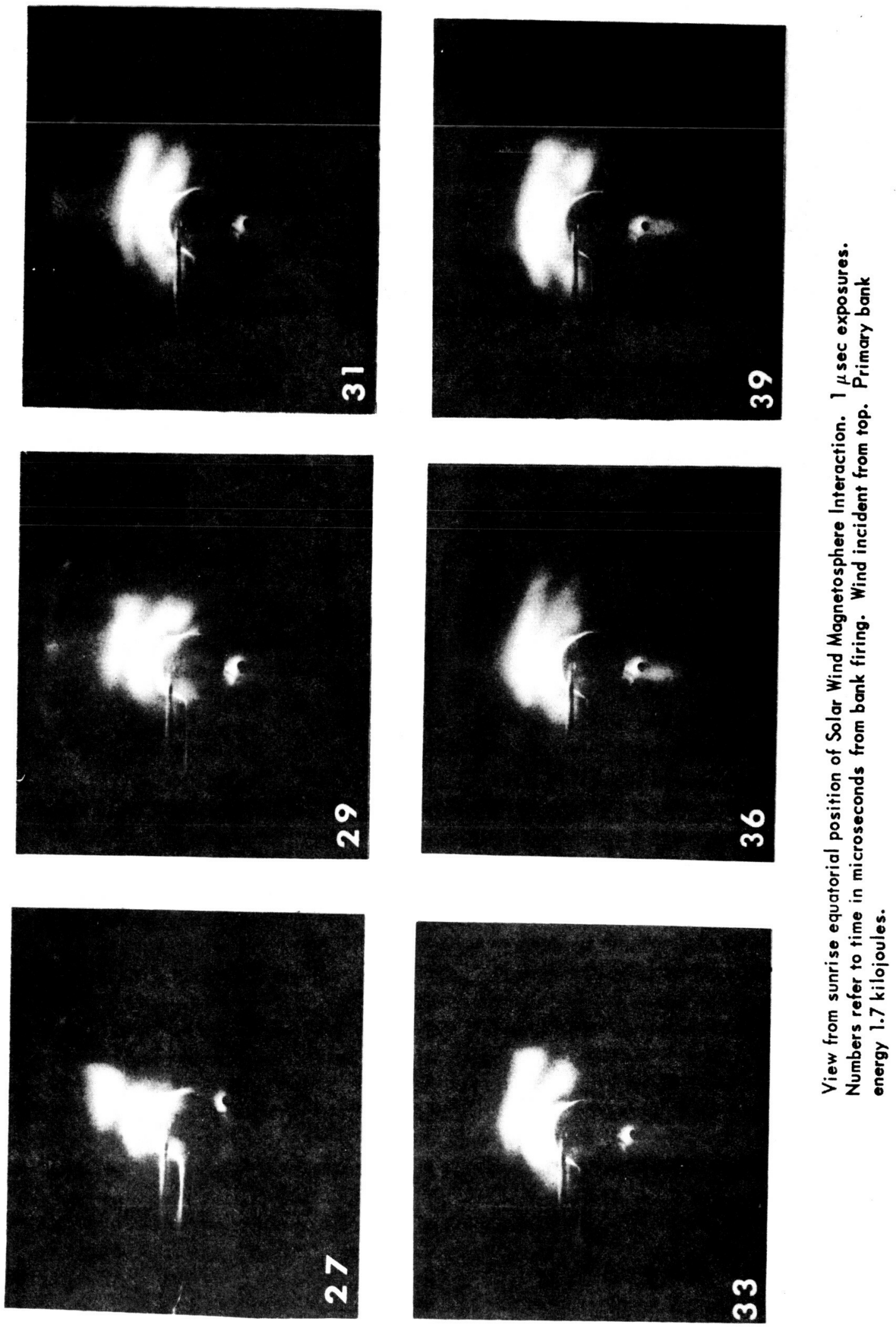


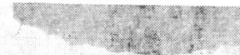
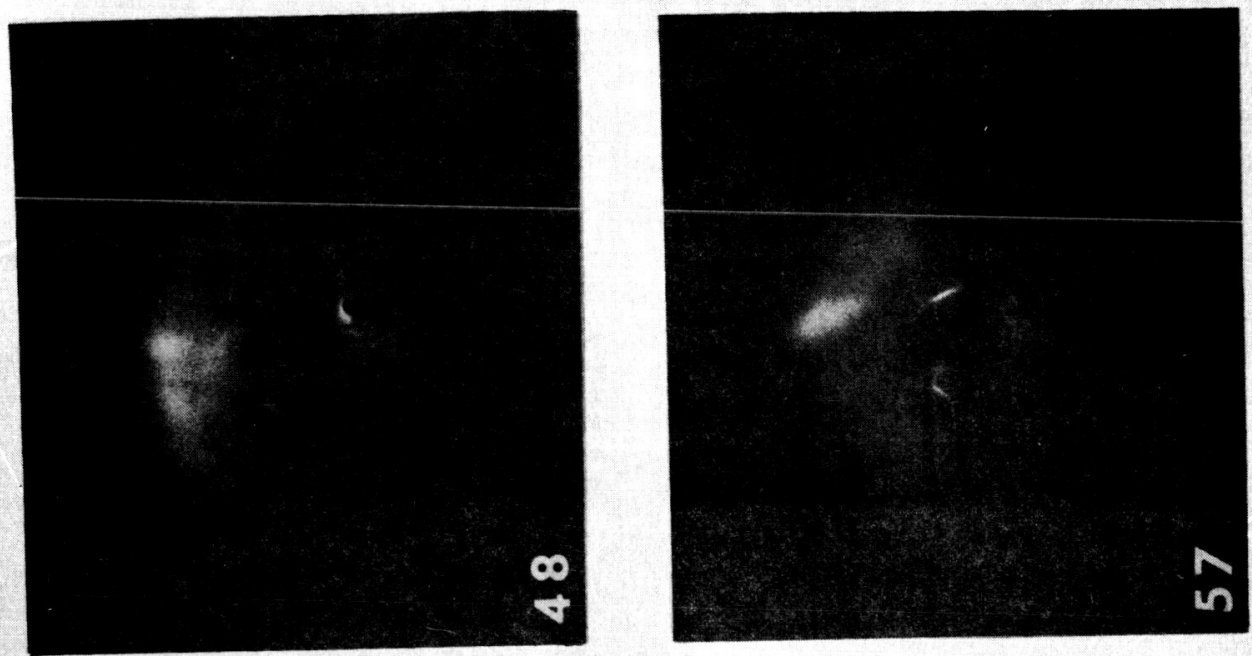

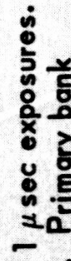
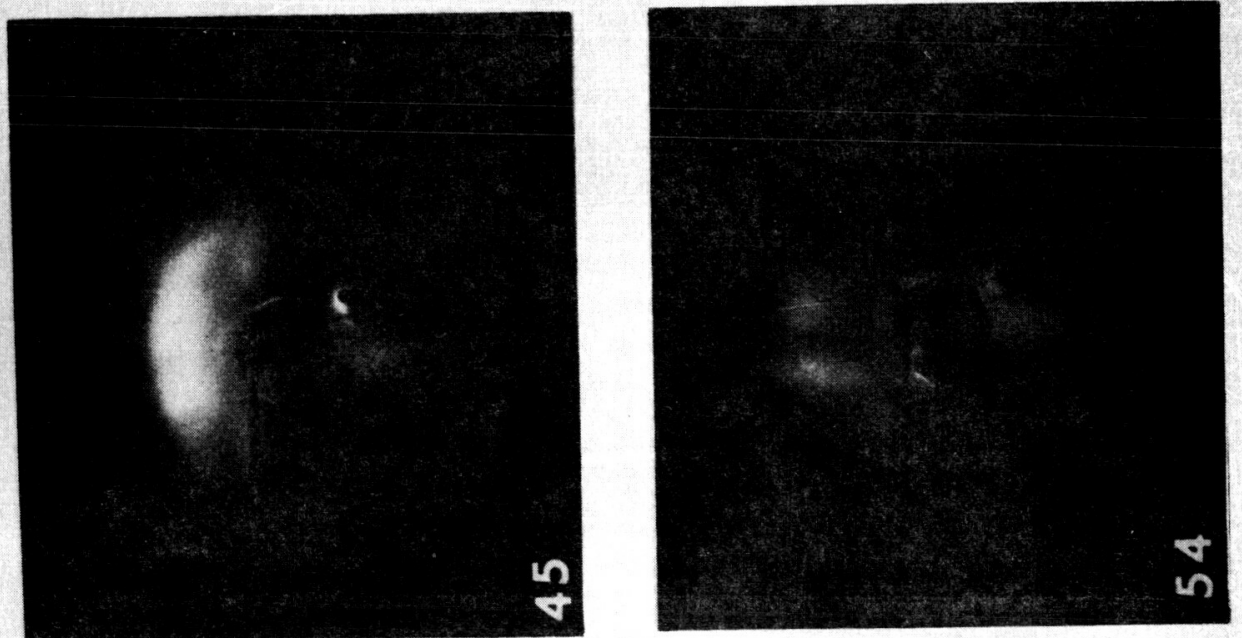

ธํํㅇ

온

흐

은

동든

ํํㄴ

둥

这

ह

흥

동

n

n
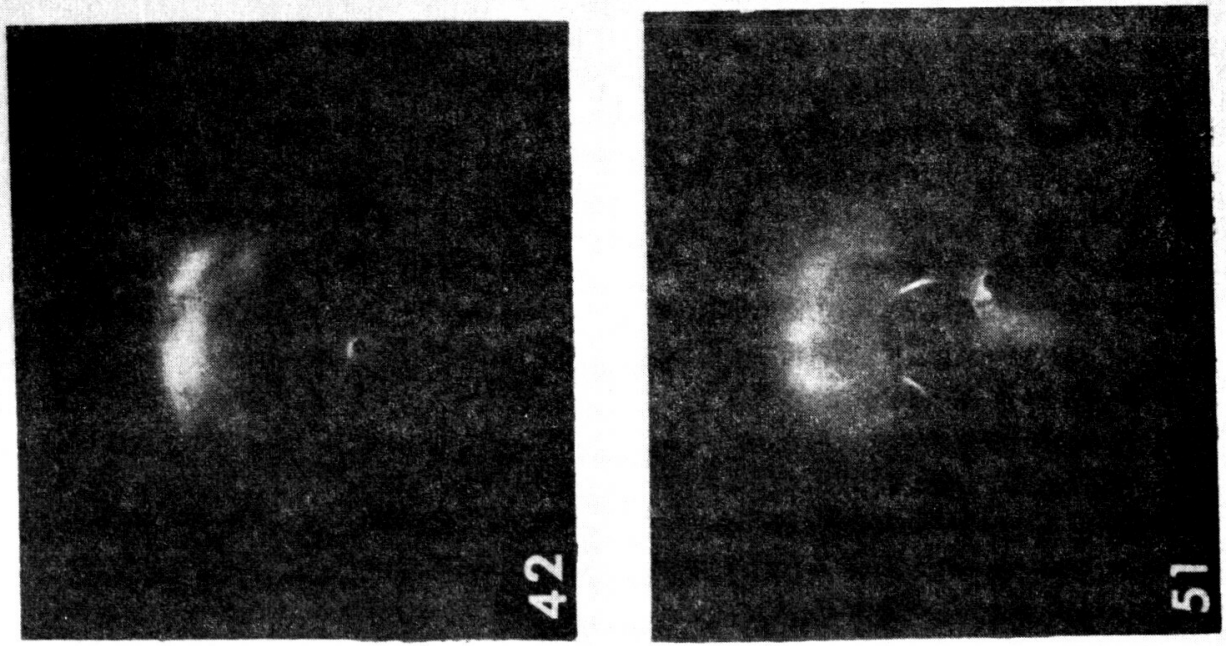

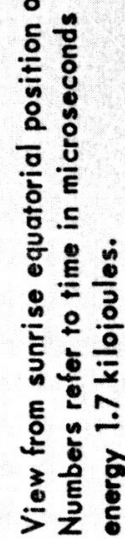




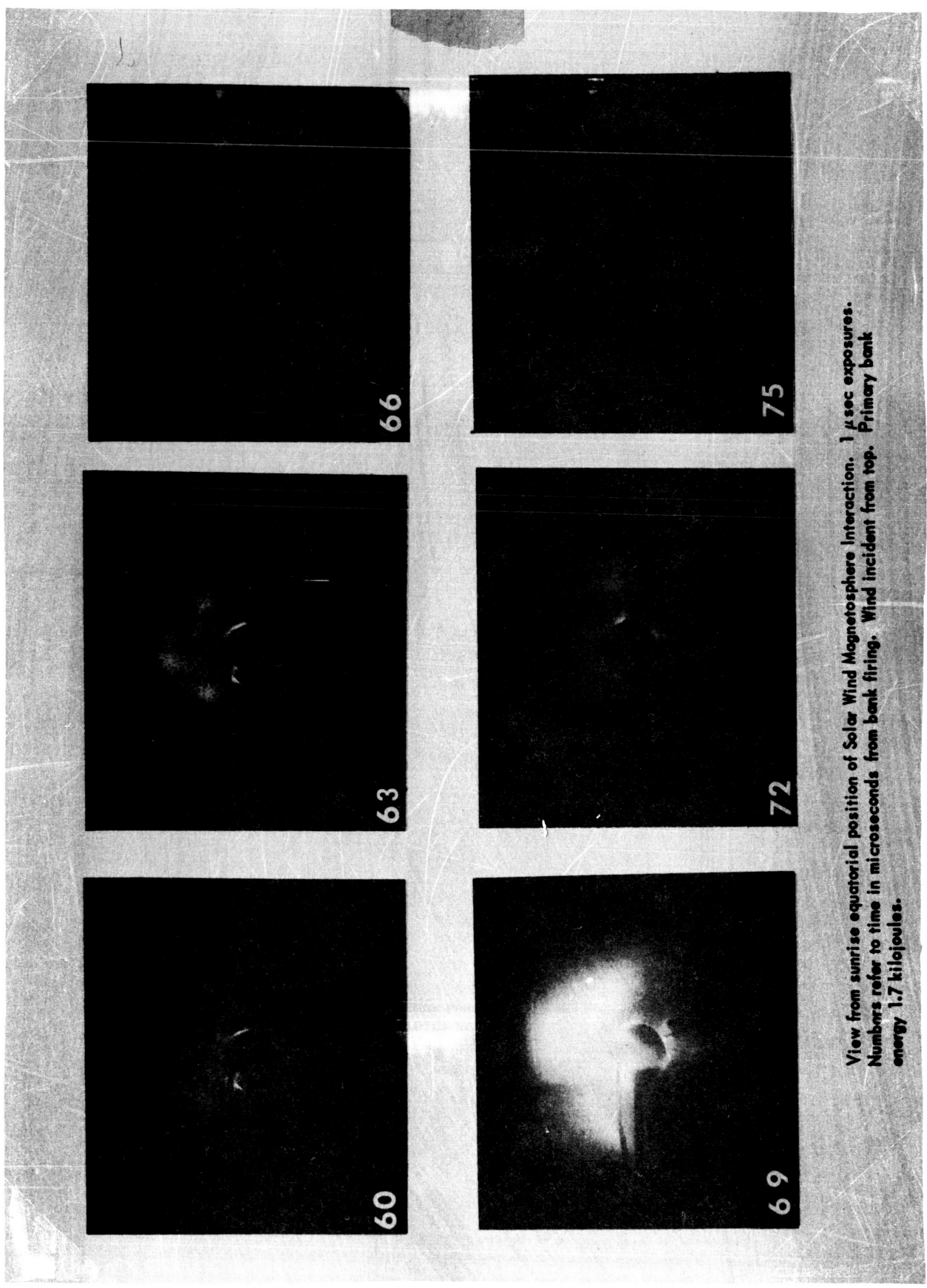




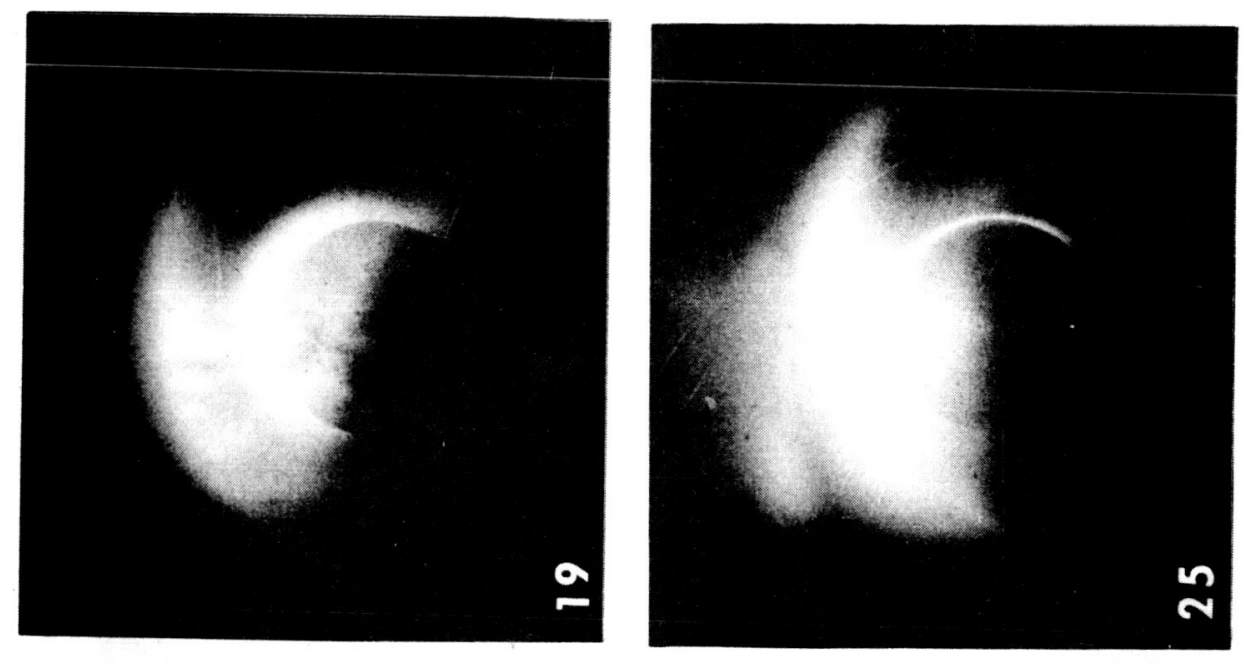

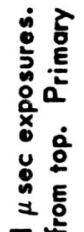
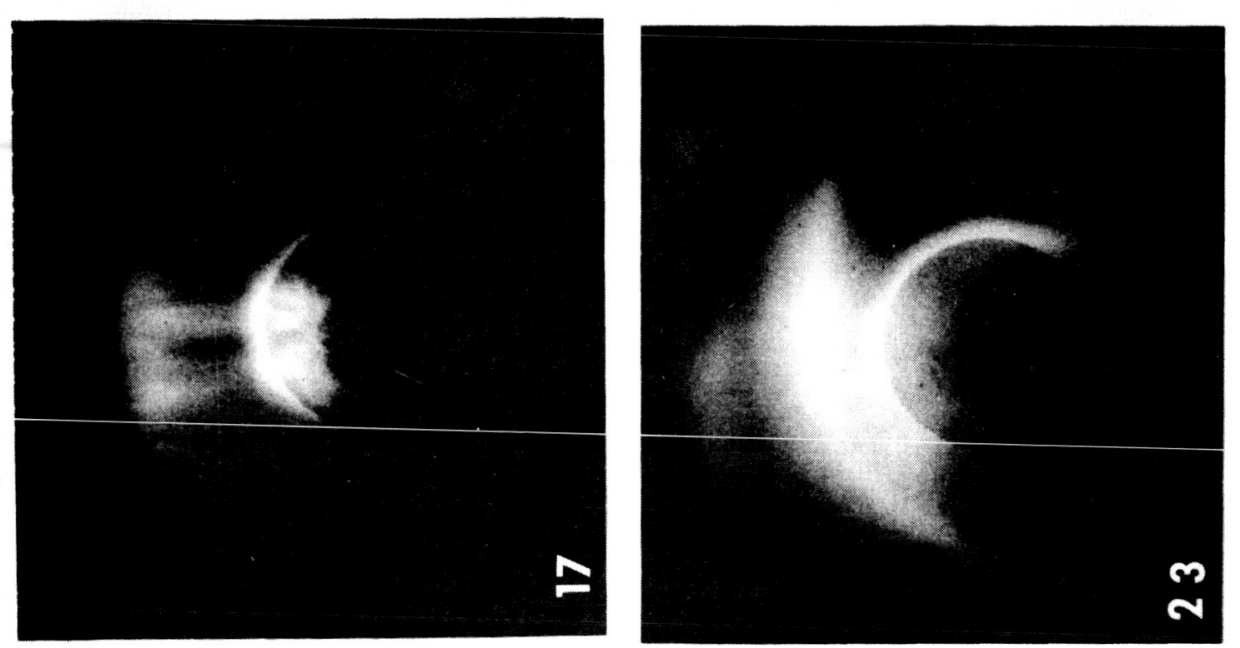

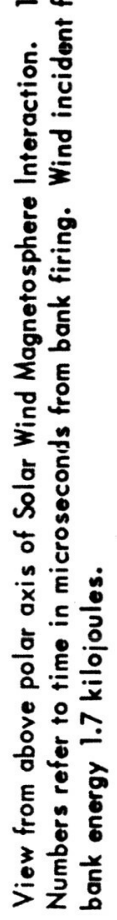
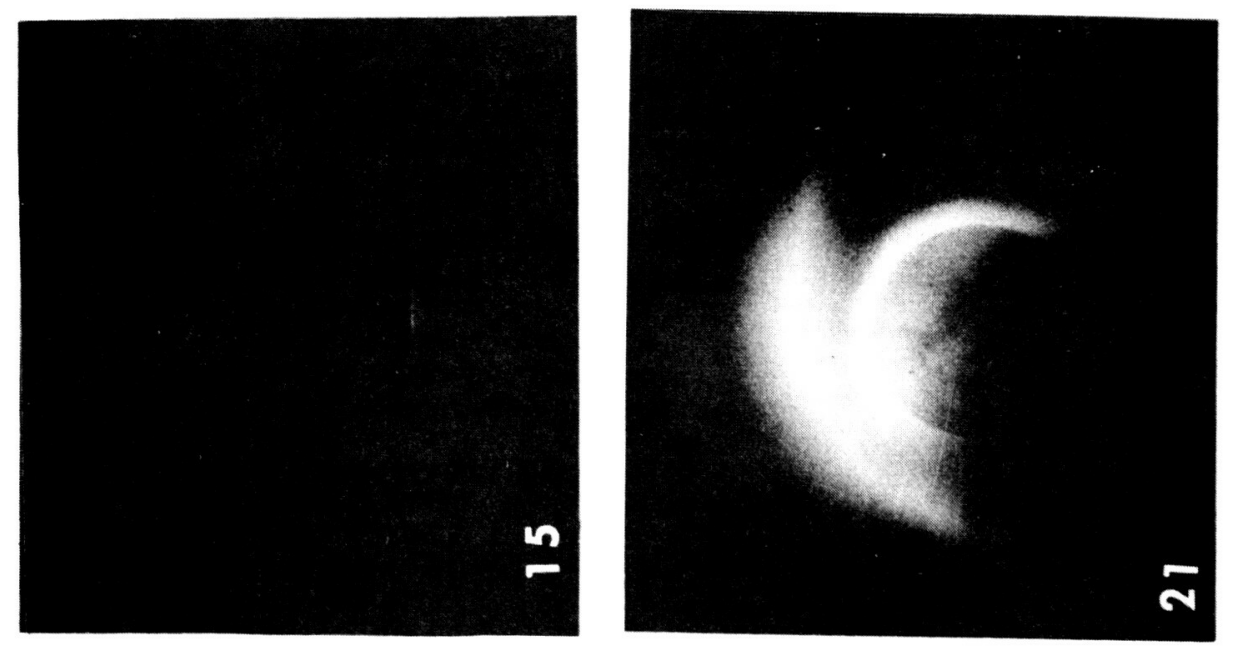

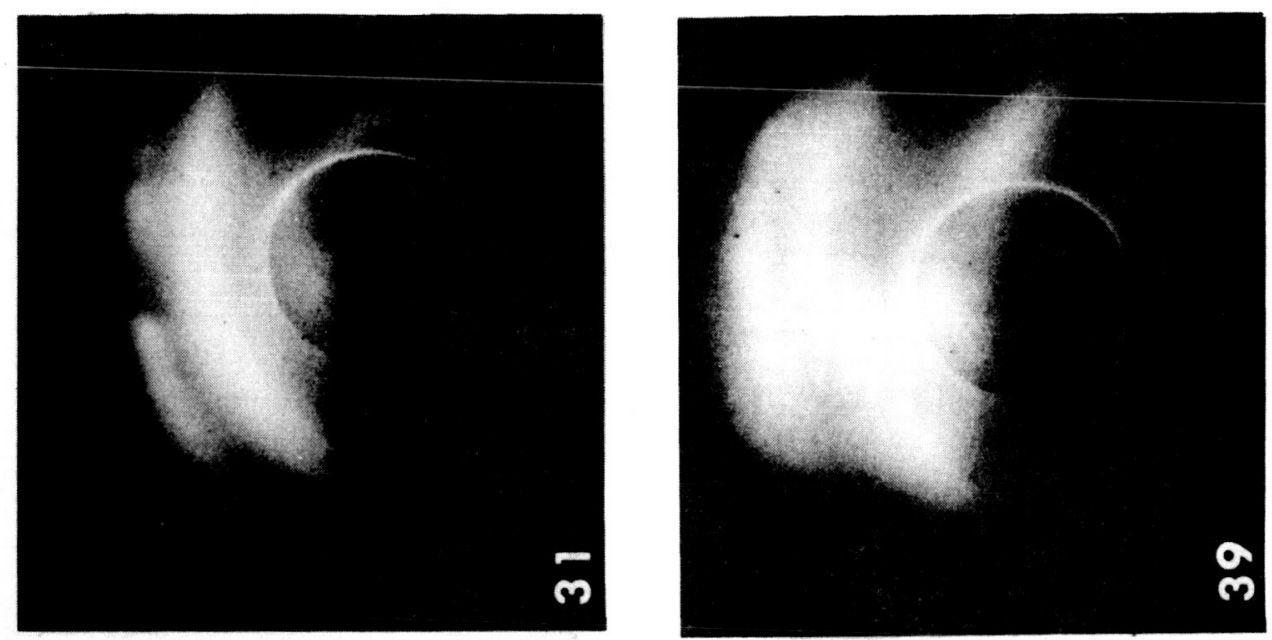

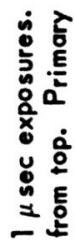
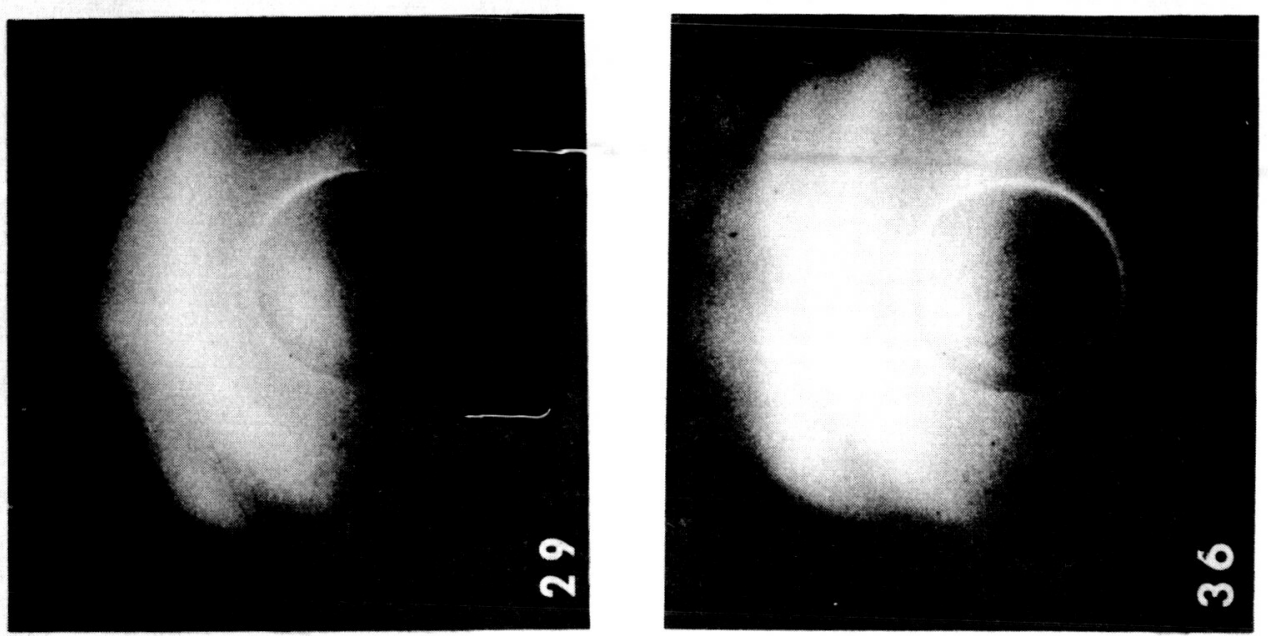

ف든

웅

눙

동

톤

눈

흥

\&ั

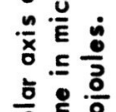

흥 을 응

웃

ㅇํㅇ

톤

方莘
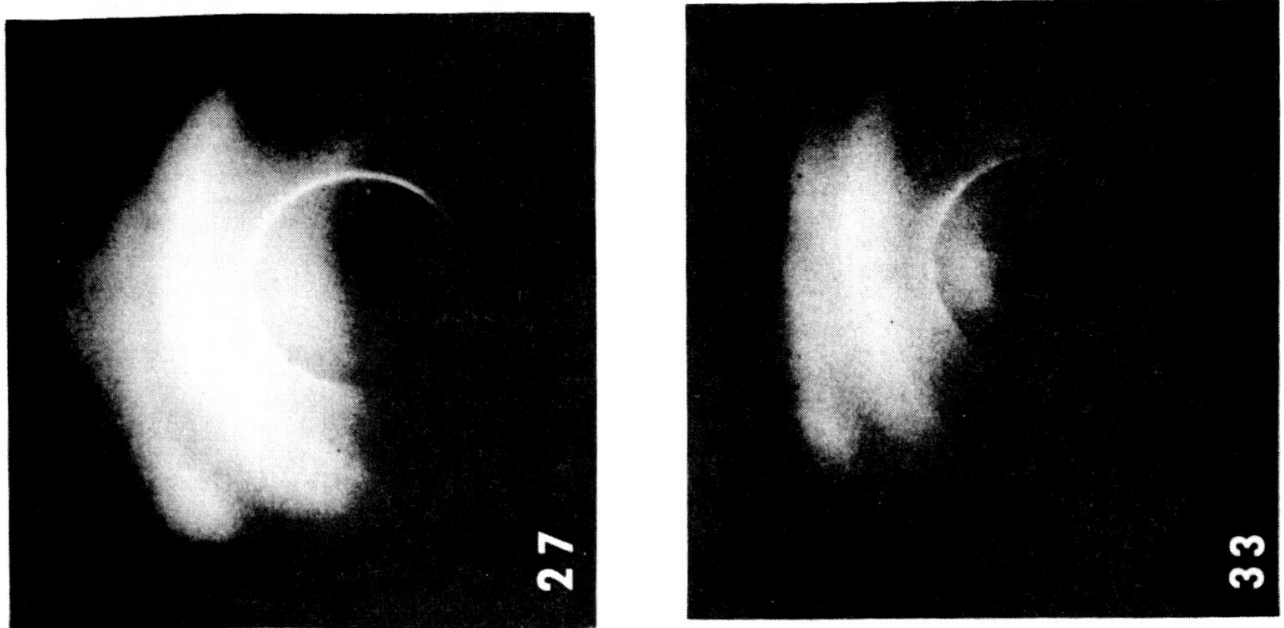

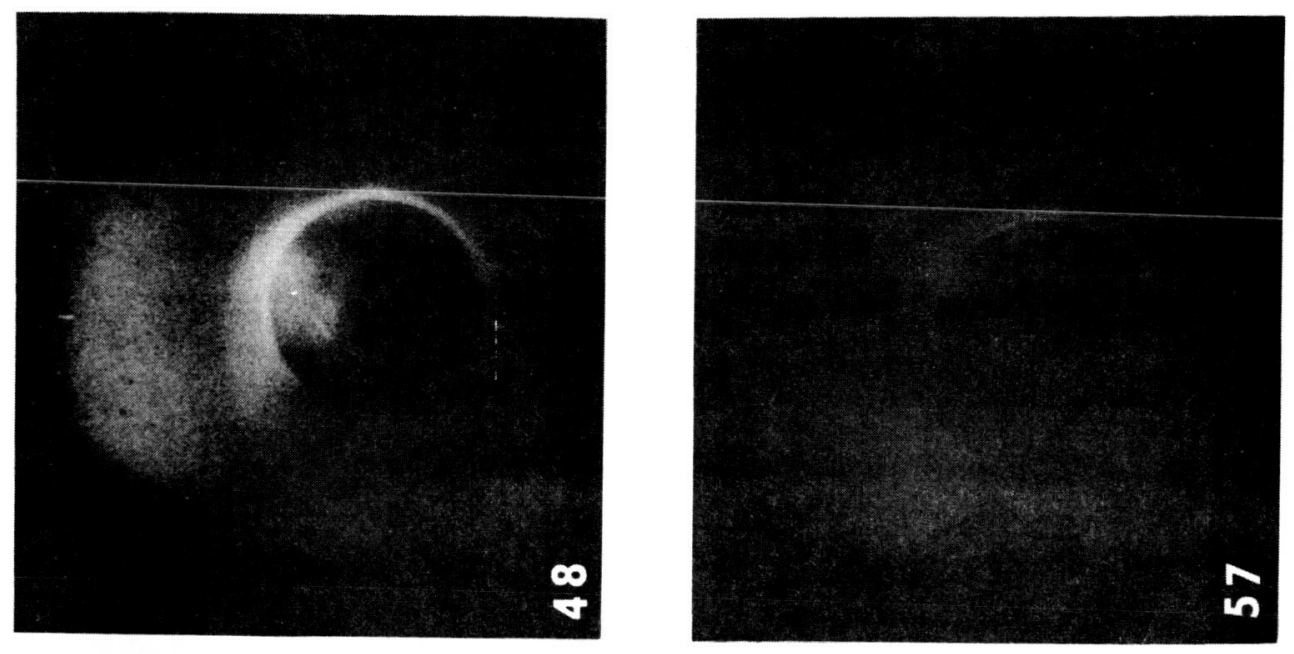

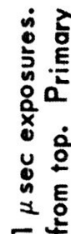
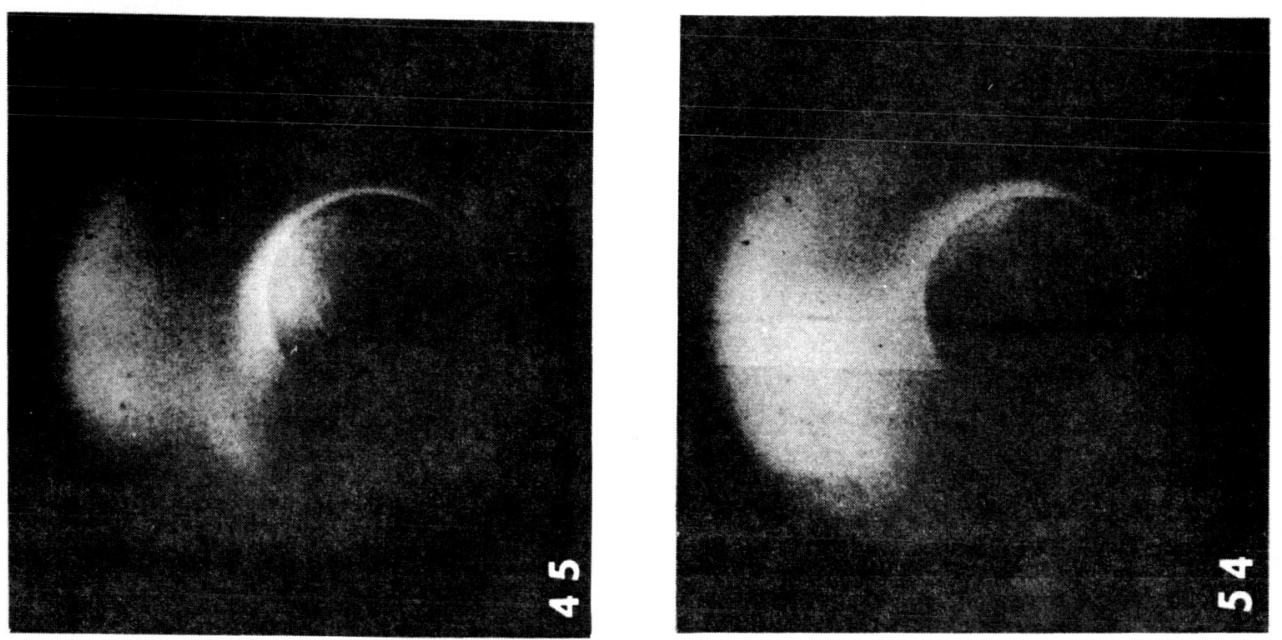

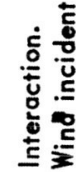

은.

동

훈

둔

흥

항

吕定它

흥 톨 $\frac{0}{3}$

$\therefore$ 음

응 -

E

站完
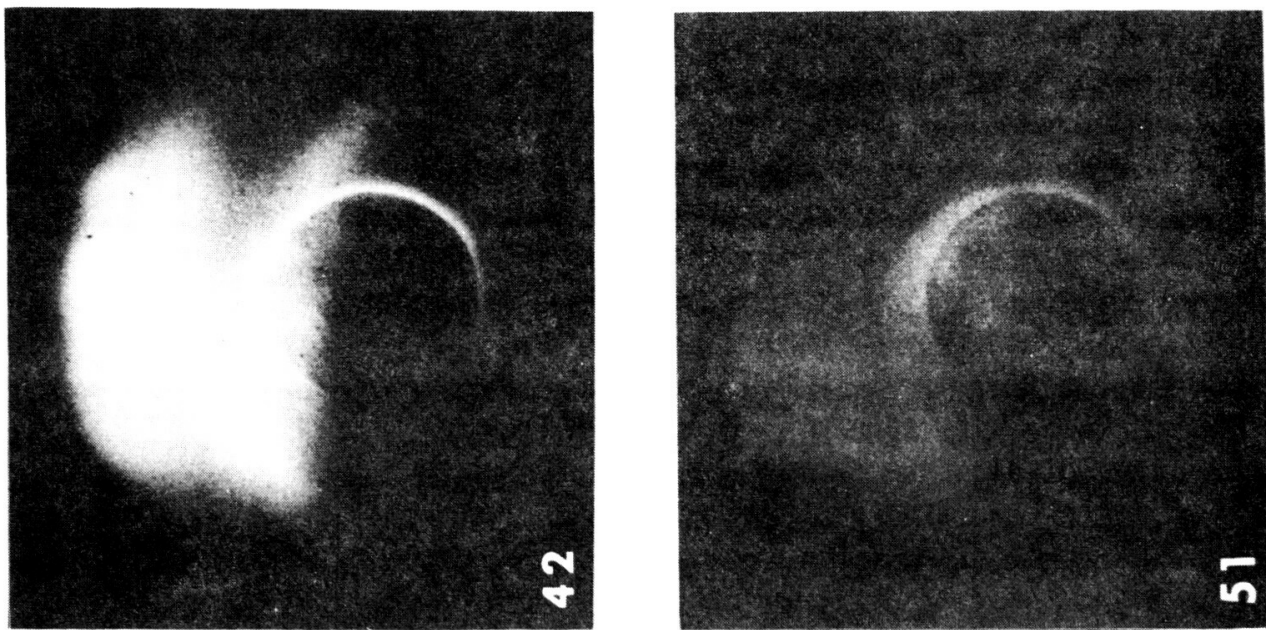


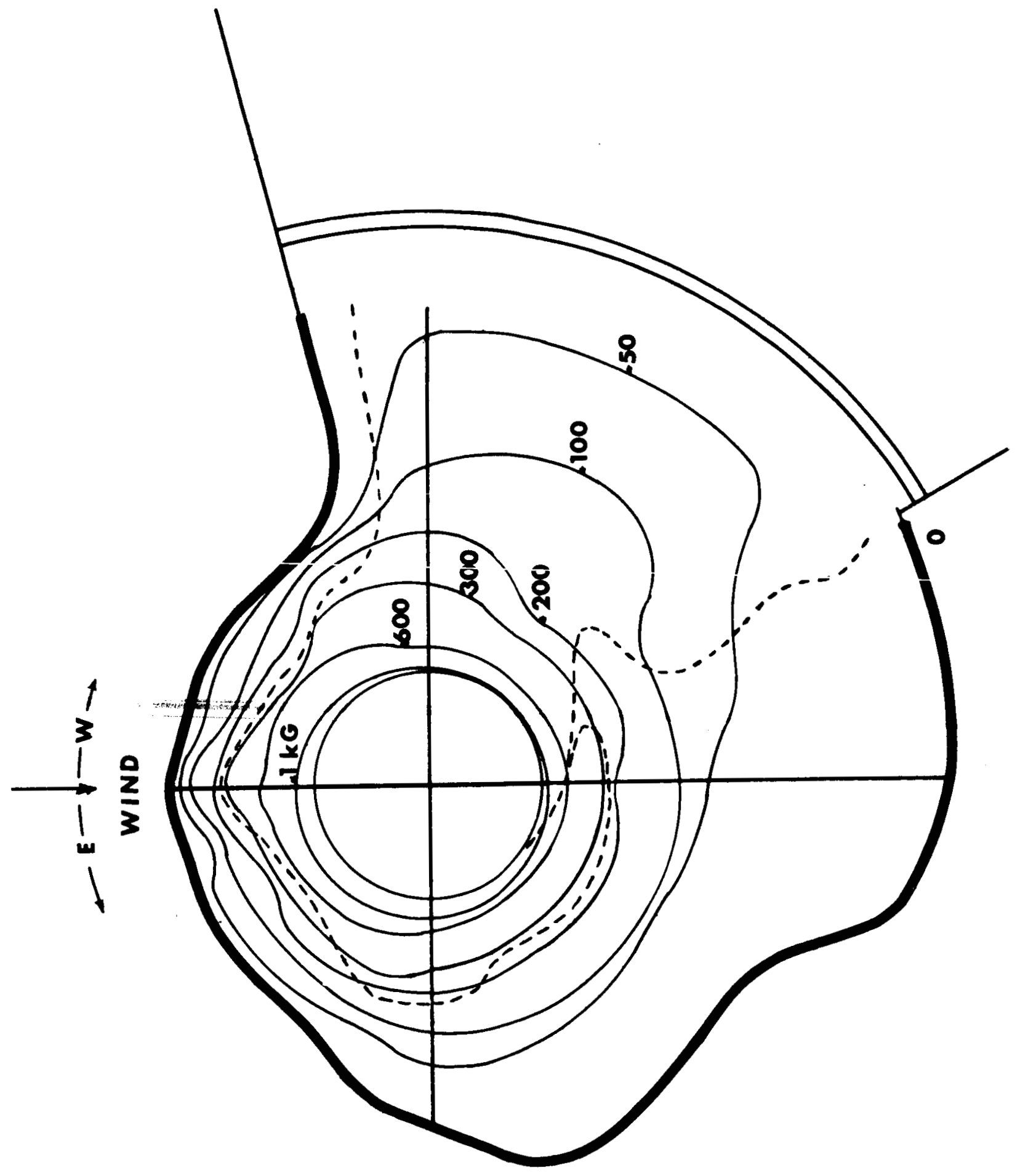

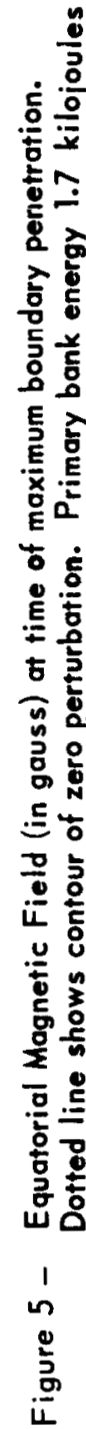

